\title{
Molecular mechanisms of endothelial to mesenchymal cell transition (EndoMT) in experimentally induced fibrotic diseases
}

\author{
Sonsoles Piera-Velazquez, Sergio A Jimenez \\ From Fibroproliferative disorders: from biochemical analysis to targeted therapies \\ Frauenchiemsee, Germany. 25-30 September 2010
}

\begin{abstract}
Several recent studies have demonstrated that endothelial to mesenchymal transition (EndoMT), a newly recognized type of cellular transdifferentiation may be an important source of myofibroblasts during the development of experimentally induced pulmonary, cardiac and kidney fibrosis. EndoMT is a complex biological process induced by members of the transforming growth factor (TGF- $\beta$ ) family of regulatory polypeptides in which endothelial cells adopt a mesenchymal or myofibroblastic phenotype acquiring motile and contractile properties and initiating expression of mesenchymal cell products such as $\alpha$ smooth muscle actin ( $\alpha$-SMA) and type I collagen. Although these experimental studies provide compelling evidence for the participation of EndoMT in the development of experimentally-induced fibrotic processes the precise role of EndoMT in the pathogenesis of human fibrotic disorders requires confirmation and validation from studies of human clinical pathologic conditions. Such confirmation should lead to a change in the paradigm of the origin of profibrogenic myofibroblasts involved in human fibrotic diseases. Further understanding of the molecular mechanisms and the regulatory pathways involved in EndoMT may lead to the development of novel therapeutic approaches for the incurable and often devastating fibrotic disorders.
\end{abstract}

\section{Introduction}

It is generally accepted that activated mesenchymal cells or myofibroblasts play a crucial role in the pathogenesis of various fibrotic diseases including interstitial pulmonary fibrosis, systemic sclerosis and liver or cardiac fibrosis being responsible for the exaggerated production and accumulation of extracellular matrix proteins in various organs affected by these diseases [1-4]. Although the etiologic factors that initiate the fibrotic diseases are diverse and in most instances remain unknown, the accumulation of activated myofibroblasts in affected tissues and the persistence of their elevated biosynthetic functions are crucial determinants of the severity and rate of progression of these diseases, and of their clinical course, response to therapy, prognosis, and mortality. Thus, a precise understanding of the origin of these cells and of

* Correspondence: sergio.jimenez@jefferson.edu

Jefferson Institute of Molecular Medicine, Thomas Jefferson University, Philadelphia, PA 19107, USA the mechanisms involved in the regulation of their intricate functions are of paramount importance for the development of effective therapeutic approaches for the vast spectrum of disorders associated with tissue and organ fibrosis [5,6]. Myofibroblasts in the fibrotic diseases are derived from at least three sources: 1) expansion and activation of resident tissue fibroblasts $[7,8] ; 2)$ transition of epithelial cells into mesenchymal cells, a process known as epithelial-mesenchymal transition [9-14]; and 3) tissue migration of bone marrow-derived circulating fibrocytes $[15,16]$. Recently, endothelial to mesenchymal transition (EndoMT), a newly recognized type of cellular transdifferentiation [17], has emerged as another possible source of tissue myofibroblasts which may play a crucial role in the pathogenesis of fibrotic diseases $[18,19]$. EndoMT is a complex biological process in which endothelial cells lose their specific endothelial cell markers, such as vascular endothelial (VE) cadherin, and acquire a mesenchymal or myofibroblastic phenotype 
initiating expression of mesenchymal cell products including $\alpha$-smooth muscle actin ( $\alpha$-SMA), vimentin, and types I and III interstitial collagens. Besides acquisition of an activated pro-fibrogenic phenotype these cells also become motile and are capable of migrating into surrounding tissues. Although in the past EndoMT was believed to be a rare phenomenon confined to certain stages of embryonic development $[17,20]$ its occurrence in fibrotic disorders is gaining increased attention. Indeed, multiple antibody immunofluorescence confocal microscopy studies and endothelial cell lineage analyses during the development of various experimentallyinduced animal models of tissue fibrosis have demonstrated the participation of EndoMT in the pathogenesis of fibrotic processes in various organs [21-26]. Although numerous studies have examined the role of epithelial mesenchymal transition (EMT) in the pathogenesis of fibrotic disorders [27] and there has been extensive investigation of the molecular events responsible for this process [28-31], studies examining the mechanisms involved in EndoMT and its potential participation in pathologic tissue fibrosis in human diseases are limited.

\section{EndoMT in experimentally-induced organ fibrosis}

The occurrence of EndoMT in experimentally induced cardiac fibrosis was originally described by Zeisberg et al. [21] employing endothelial cell lineage analysis in transgenic mice. In these studies, analyses of the proportion of fibroblasts present in the fibrotic myocardium of mice with aortic banding induced myocardial fibrosis showed that from 27 to $35 \%$ of fibroblasts originated from endothelial cells. Several other studies have confirmed the emergence of activated fibroblasts originating from endothelial cells in various experimentally induced models of cardiac fibrosis $[22,23]$ and collectively have suggested that in these experimental conditions EndoMT represents an important contributor to the generation of fibrotic tissue and, therefore, this pathway may represent a novel therapeutic target. EndoMT has also emerged as a potentially important mechanism in the development and progression of experimentally induced pathological kidney and pulmonary fibrosis. Numerous studies have shown that EndoMT is a novel pathway leading to fibrotic development in diabetic nephropathy and other models of kidney fibrosis. An extensive study by Zeisberg et al. [24] examined the role of EndoMT in three murine models of chronic kidney disease: unilateral ureteral obstructive nephropathy, streptozotocin-induced diabetic nephropathy and a model of Alport renal disease. The results of these studies indicated that $30-50 \%$ of myofibroblasts in the fibrotic kidneys, identified by their expression of a fibroblast phenotype and $\alpha$-SMA, display the endothelial cell specific CD31 surface marker indicating their endothelial cell origin. These studies were validated by endothelial cell lineage tracing and were also confirmed by studies from other laboratories $[18,25]$. The possible role of EndoMT in experimentally induced pulmonary fibrosis was examined by Hashimoto et al. [26]. These authors evaluated EndoMT as a source of interstitial fibroblasts in bleomycin-induced lung fibrosis using double-transgenic mice in which LacZ was stably expressed in endothelial cells and therefore allowed the histological identification of any cells originated from an endothelial cell lineage. Following endotracheal injection of bleomycin the areas of fibrotic involvement were shown to contain large numbers of fibroblasts of endothelial origin. To directly demonstrate the presence of endothelial cell-derived lung fibroblasts in affected lung fibrotic tissues, lung fibroblasts were isolated and cultured from either saline injected control mice or from mice that received bleomycin injections. These studies revealed that approximately $16 \%$ of lung fibroblasts in the cultures from bleomycin-treated mice were derived from endothelial cells as illustrated in Figure 1.

\section{EndoMT of human endothelial cells}

In contrast with the extensive evidence from experimental animal models demonstrating the important participation of EndoMT in the development of tissue fibrosis studies with human endothelial cells are just beginning to appear. One such study [32] examined the conversion of human microvascular intestinal endothelial cells into mesenchymal cells following exposure to a combination of proinflammatory cytokines (TGF- $\beta$, TNF- $\alpha$ and IL-1 $\beta$ ) in vitro. The treated cells displayed a progressive loss and the eventual disappearance of endothelial-specific cell markers (CD-31 and VE-cadherin) and a parallel acquisition of mesenchymal cell markers including vimentin, collagen I and N-cadherin. These results suggested that intestinal endothelial cells exposed to an inflammatory environment may participate in the intestinal fibrotic process which accompanies intestinal inflammatory diseases. The in vitro observations were confirmed by studies of molecular co-localization in human colonic mucosa [32].

Another study examined the expression of cell surface markers specific for endothelial cells in mesenchymal/ fibroblastic cells present in the subendothelial compartment of small vessels in lungs from patients with Systemic Sclerosis-associated pulmonary fibrosis [33]. Confocal microscopy demonstrated the expression of endothelial cell markers (CD-31 and CD-34) in mesenchymal cells embedded within the subendothelial neointima of small pulmonary arteries in lung specimens from patients with Systemic Sclerosis. These two studies provide strong support to the concept that EndoMT may also play an important role in the pathogenesis of human fibrotic diseases. 


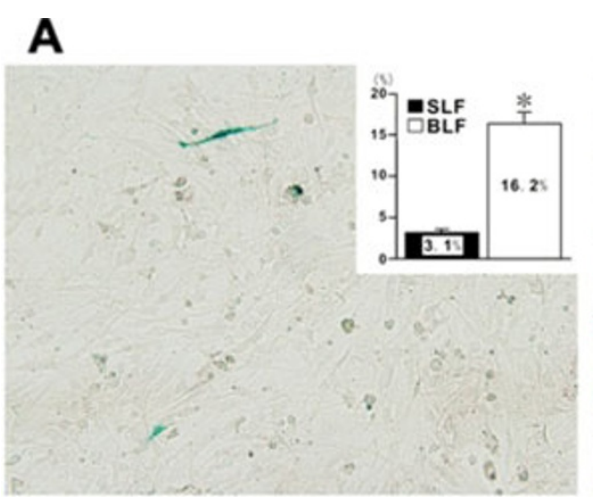

B
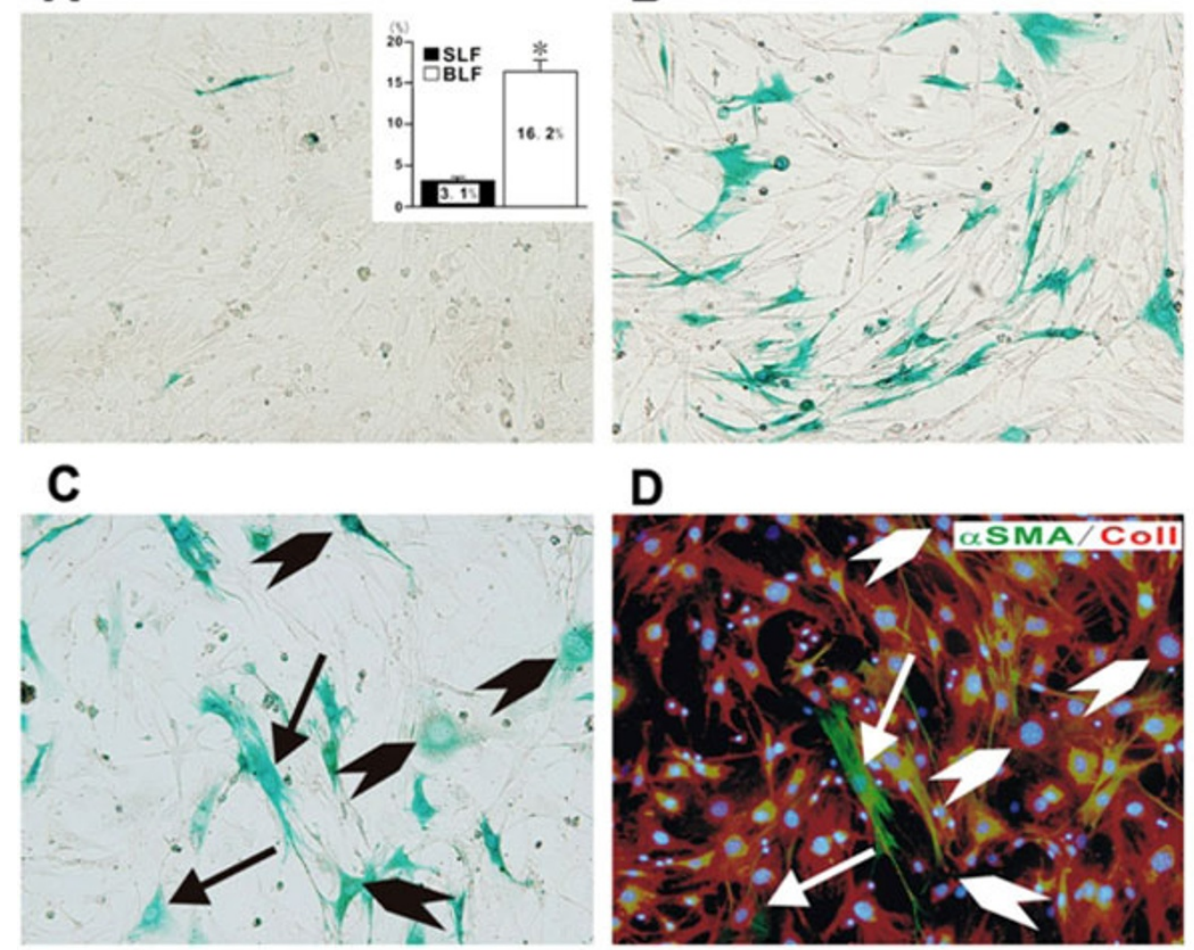

Figure 1 Demonstration of endothelial cell-derived fibroblasts in fibroblast cultures established from lung parenchyma of mice with bleomycin induced pulmonary fibrosis. Fibroblast cultures were established from lungs from mice injected intratrachealy with either normal saline or bleomycin. When the cultures reached confluency they were stained with $x$-gal to identify the cells from endothelial lineage. Note the absence of $x$-gal staining cells in the cultures from saline injected control mice (A) in contrast with the marked abundance of $x$-gal staining fibroblasts in the cultures from bleomycin injected mice (B). The inset in A shows the percentage of $x$-gal positive cells in four separate samples of cultured fibroblasts from saline injected mice (SLF) compared to eight separate samples of fibroblasts cultured from bleomycin injected mice (BLF). Figures $C$ and $D$ show sequential staining of a fibroblast culture from bleomycin injected mice with $x$-gal (C) followed by immunocytochemistry for the mesenchymal cell markers type I collagen (red) and $\alpha$ - SMA (green). The arrows indicate cells positive for $x$-gal, type I collagen, and $\alpha$-SMA, whereas the arrowheads indicate cells positive for $x$-gal and type I collagen. Reproduced from reference 26 with permission.

\section{Molecular Mechanisms of EndoMT}

In contrast to the very extensive studies conducted to unravel the molecular mechanisms and the regulatory pathways that modulate epithelial mesenchymal transition [27-31], the mechanisms involved in the EndoMT process have not been explored in detail. Few studies have examined the biochemical and molecular changes occurring in endothelial cells during their transdifferentiation into mesenchymal myofibroblasts and the regulatory events involved in this process. It is generally accepted that TGF- $\beta$ plays a crucial role in tissue fibrosis and is implicated in the pathogenesis of numerous fibrotic disorders [5,6,34-37]. Recent studies have shown that besides causing a potent stimulation of the expression of numerous genes participating in the fibrotic process, TGF- $\beta$ is involved in the generation of myofibroblasts through EndoMT [21,22,38-42]. The studies in experimentally induced cardiac hypertrophy provided an elegant demonstration of the role of TGF- $\beta$ in this process
[21]. These studies showed that TGF- $\beta$ was a crucial mediator causing endothelial cells to undergo EndoMT since there was a significant reduction in the number of mesenchymal cells originating from endothelial cells when the TGF- $\beta$ response was blunted by the deficiency of Smad3 in Smad 3+/- transgenic mice. These results have been confirmed employing a TGF- $\beta$ receptor kinase inhibitor which inhibits activated TGF- $\beta$ [40] as well as several small molecule inhibitors of intracellular phosphorylation reactions [41,42].

Besides TGF- $\beta$ it has been shown that endothelin-1 (ET1) may also participate in EndoMT. In one study, Widyantoro et al. [23] showed that endothelial cell-derived ET-1 promotes cardiac fibrosis and heart failure in diabetic hearts through stimulation of EndoMT. These features were abolished in hearts from transgenic mice with endothelial cell specific ET-1 deletion. In another study it was shown that in vitro treatment of murine pulmonary endothelial cells with ET-1 although not capable of initiating EndoMT by 
itself could excert a powerful synergistic effect with TGF- $\beta$ (Li and Jimenez; unpublished observations).

Although there are numerous studies which have conclusively shown the crucial involvement of members of the TGF- $\beta$ family of growth factors in initiation of EndoMT, the detailed molecular events and the intracellular cascades activated by TGF- $\beta$ that result in the remarkable phenotypic change of endothelial cells to mesenchymal cells have not been entirely elucidated. Recent studies in primary cultures of murine pulmonary endothelial cells [41] and in cultured human dermal microvascular cells [42] demonstrated that EndoMT involves both Smad-dependent and Smad-independent pathways. The downstream signaling pathway initiated by TGF- $\beta$ resulted in strong upregulation of the transcriptional repressor Snail1. Snail1 causes potent inhibition of E-cadherin gene transcription in cultured cells and plays an important role in the epithelial to mesenchymal transition $[27,28]$ indicating that the EndoMT process shares similar molecular mechanisms with epithelial mesenchymal transdifferentiation.

Several studies explored the underlying molecular pathways that may cause the significant loss of endothelial-specific markers while inducing strong de novo mesenchymal phenotypes. These studies identified the c-Abl protein kinase (c-Abl), protein kinase $\mathrm{C} \delta(\mathrm{PKC}-\delta)$, and glycogen synthase kinase $3 \beta$ (GSK-3 $\beta$ ) as important participants and that GSK-3 $\beta$ kinase phosphorylation was a crucial event in this process $[41,42]$. It is well known that phosphorylation of specific serine residues in GSK$3 \beta$ results in inactivation of the kinase which in turn induces the nuclear accumulation of Snaill followed by a profound increase in the expression of its corresponding gene. The transcriptional effects of Snail1 induce the expression of a mesenchymal cell-specific phenotype although the precise mechanisms involved remain obscure. In contrast, in the absence of GSK-3 $\beta$ phosphorylation the GSK- $3 \beta$ kinase is active and induces the proteosomal degradation of Snaill, thus abrogating the acquisition by endothelial cells of a mesenchymal cell phenotype. Other studies have shown that several important regulatory pathways including the canonical Wnt pathway, the HIF-1 $\alpha$ hypoxia induced pathway, and the response to cellular stress may also participate in the regulation of EndoMT [43,44] as illustrated in Figure 2.

In summary, numerous recent studies have provided strong evidence for the prominent participation of EndoMT in the generation of activated myofibroblasts during the development of experimentally induced tissue fibrosis suggesting that EndoMT plays a crucial role in fibrotic diseases. There is some early experimental evidence providing support and validation for the participation of EndoMT in the development of human intestinal fibrosis and of Systemic Sclerosis-associated pulmonary

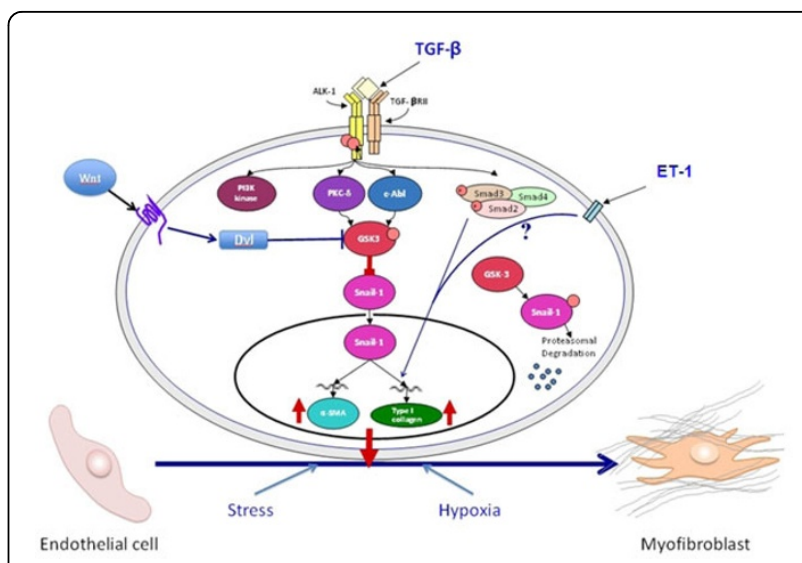

Figure 2 Schematic diagram showing the putative TGF- $\beta$ signaling pathways involved in EndoMT. The diagram shows the numerous putative pathways that may participate in the EndoMT process and may be involved in the pathogenesis of human fibrotic disorders. One central pathway initiated following ligand-binding activation of the Smad-independent TGF- $\beta$ pathway causes phosphorylation of GSK-3 $\beta$ mediated by PKC- $\delta$ and the CAbl nonreceptor kinase. Phosphorylation of GSK-3 $\beta$ at serine 9 (ser9) causes its inhibition which then allows Snail1 to enter the nucleus. Nuclear accumulation of Snail1 results in marked stimulation of Snail1 expression which then leads to acquisition of the myofibroblast phenotype with stimulation of $\alpha$-SMA. The inhibition of GSK-3 $\beta$ ser9 phosphorylation by specific inhibition of PKC- $\delta$ or c-Abl activity allows GSK-3 $\beta$ to phosphorylate Snail1 targeting it for proteosomal degradation and thus, effectively abolishes the acquisition of the myofibroblastic phenotype and the fibrotic response. Other pathways such as the ET-1, Wnt, hypoxia and cellular stress pathways may also participate although the molecular events have not been fully elucidated. Modified from Li and Jimenez [37].

fibrosis. The firm demonstration of the occurrence of EndoMT in human fibrotic diseases and further understanding of the molecular mechanisms involved may lead to the pharmacologic modulation or abrogation of this pathway in human fibrotic disorders and may represent a novel therapeutic approach for these devastating diseases.

\section{Acknowledgements}

This article has been published as part of Fibrogenesis \& Tissue Repair Volume 5 Supplement 1, 2012: Proceedings of Fibroproliferative disorders: from biochemical analysis to targeted therapies. The full contents of the supplement are available online at http://www.fibrogenesis.com/ supplements/5/S1.

Supported by NIH Grant 5 R01 AR19616 to Sergio A. Jimenez. The expert assistance of Melissa Bateman in the preparation of the manuscript is gratefully acknowledged.

\section{Competing interests}

The authors declare that they have no competing interests.

Published: 6 June 2012

\section{References}

1. Hinz B, Gabbiani G: Fibrosis: recent advances in myofibroblast biology and new therapeutic perspectives. F1000 Biol Rep 2010, 11:2-78. 
2. Neilson EG: Mechanisms of disease: Fibroblasts-a new look at an old problem. Nat Clin Pract Nephrol 2006, 2:101-8.

3. Abraham DJ, Eckes B, Rajkumar V, Krieg T: New developments in fibroblast and myofibroblast biology: implications for fibrosis and scleroderma. Curr Rheumatol Rep 2007, 9:136-43.

4. Heinz B, Phan SH, Thannickal VJ, Galli A, Bochaton-Piallat ML, Gabbiani G: The myofibroblast: one function, multiple origins. Am J Pathol 2007, 170:1807-16

5. Rosenbloom J, Castro SV, Jimenez SA: Narrative review: fibrotic diseases: cellular and molecular mechanisms and novel therapies. Ann Intern Med 2010, 152:159-66.

6. Wei J, Bhattacharyya S, Tourtellotte WG, Varga J: Fibrosis in systemic sclerosis: Emerging concepts and implications for targeted therapy. Autoimmun Rev 2010.

7. McAnulty RJ: Fibroblasts and myofibroblasts: their source, function and role in disease. Int J Biochem Cell Biol 2007, 39:666-71.

8. Postlethwaite AE, Shigemitsu H, Kanangat S: Cellular origins of fibroblasts: possible implications for organ fibrosis in systemic sclerosis. Curr Opin Rheumatol 2004, 16:733-8.

9. Thiery JP, Acloque H, Huang RY, Nieto MA: Epithelial-mesenchymal transitions in development and disease. Cell 2009, 139:871-90

10. Kalluri R, Neilson EG: Epithelial-mesenchymal transition and its implications for fibrosis. J Clin Invest 2003, 112:1776-84.

11. Willis BC, Borok Z: TGF-beta-induced EMT: mechanisms and implications for fibrotic lung disease. Am J Physiol Lung Cell Mol Physiol 2007, 293. L525-34.

12. Willis $B C$, duBois RM, Borok Z: Epithelial origin of myofibroblasts during fibrosis in the lung. Proc Am Thorac Soc 2006, 3:377-82.

13. Rastaldi MP: Epithelial-mesenchymal transition and its implications for the development of renal tubulointerstitial fibrosis. J Nephrol 2006, 19:407-12.

14. Zeisberg M, Kalluri R: Fibroblasts emerge via epithelial-mesenchymal transition in chronic kidney fibrosis. Front Biosci 2008, 13:6991-8.

15. Herzog EL, Bucala R: Fibrocytes in health and disease. Exp Hematol 2010, 38:548-56

16. Bellini $A$, Mattoli $S$ : The role of the fibrocytes, a bone marrow-derived mesenchymal progenitor, in reactive and reparative fibroses. Lab Invest 2007, 87:858-70.

17. Arciniegas $E$, Sutton $A B$, Allen TD, Schor AM: Transforming growth factor beta 1 promotes the differentiation of endothelial cells into smooth muscle-like cells in vitro. J Cell Sci 1992, 103:521-9.

18. Li J, Bertram JF: Review: Endothelial-myofibroblast transition, a new player in diabetic renal fibrosis. Nephrology (Carlton) 2010, 15:507-12.

19. Piera-Velazquez S, Li Z, Jimenez SA: Role of Endothelial-Mesenchymal Transition (EndoMT) in the Pathogenesis of Fibrotic Disorders. Am J Pathol 2011, 179:1074-1084

20. Arciniegas E, Neves CY, Carrillo LM, Zambrano EA, Ramirez R: Endothelialmesenchymal transition occurs during embryonic pulmonary artery development. Endothelium 2005, 12:193-200.

21. Zeisberg EM, Tarnavski O, Zeisberg M, Dorfman AL, McMullen JR, Gustafsson E, Chandraker A, Yuan X, Pu WT, Roberts AB, Neilson EG, Sayegh MH, Izumo S, Kalluri R: Endothelial-to-mesenchymal transition contributes to cardiac fibrosis. Nat Med 2007, 13:952-61.

22. Goumans $M$, van Zonneveld AJ, ten Dijke P: Transforming growth factor $\beta$-Induced endothelial-to-mesenchymal transition: A switch to cardiac fibrosis? Trends Cardiovasc Med 2008, 18:293-8.

23. Widyantoro B, Emoto N, Nakayama K, Anggrahini DW, Adiarto S, Iwasa N, Yagi K, Miyagawa K, Rikitake Y, Suzuki T, Kisanuki YY, Yanagisawa M, Hirata K: Endothelial cell-derived endothelin-1 promotes cardiac fibrosis in diabetic hearts through stimulation of endothelial-to-mesenchymal transition. Circulation 2010, 121:2407-18.

24. Zeisberg EM, Potenta SE, Sugimoto H, Zeisberg M, Kalluri R: Fibroblasts in kidney fibrosis emerge via endothelial-to-mesenchymal transition. J Am Soc Nephrol 2008, 19:2282-7.

25. Li J, Qu X, Bertram JF: Endothelial-Myofibroblast Transition Contributes to the Early Development of Diabetic Renal Interstitial Fibrosis in Streptozotocin-Induced Diabetic Mice. Am J Pathol 2009, 175:1380-8.

26. Hashimoto N, Phan SH, Imaizumi K, Matsuo M, Nakashima H, Kawabe T, Shimokata K, Hasegawa Y: Endothelial-mesenchymal transition in bleomycin-induced pulmonary fibrosis. Am J Respir Cell Mol Biol 2010, 43:161-72
27. Nieto MA: Epithelial-mesenchymal transitions in development and disease: Old views and new perspectives. Int I Dev Biol 2009, 53:1541-1547.

28. Cano A, Perez-Moreno MA, Rodrigo I, Locascio A, Blanco MJ, del Barrio MG, Portillo F, Nieto MA: The transcription factor snail controls epithelialmesenchymal transitions by repressing E-cadherin expression. Nat Cell Biol 2000, 2:76-83.

29. Thiery JP, Sleeman JP: Complex networks orchestrate epithelialmesenchymal transitions. Nat Rev Mol Cell Biol 2006, 7:131-42.

30. Zhou BP, Deng J, Xia W, Xu J, Li YM, Gunduz M, Hung MC: Dual regulation of snail by GSK-3beta-mediated phosphorylation in control of epithelialmesenchymal transition. Nat Cell Biol 2004, 6:931-940.

31. Doble BW, Woodgett JR: Role of glycogen synthase kinase- 3 in cell fate and epithelial- mesenchymal transitions. Cells Tissues Organs 2007, 185:73-84.

32. Rieder F, Kessler SP, West GA, Bhilocha S, de la Motte C, Sadler TM, Gopalan B, Stylianou E, Fiocchi C: Inflammation-Induced Endothelial-toMesenchymal Transition A Novel Mechanism of Intestinal Fibrosis. Am J Pathol 2011, 179:2660-73.

33. Mendoza F, Piera-Velazquez S, Farber JL, Jimenez SA: Role of EndothelialMesenchymal Transition (EndoMT) in the Pathogenesis of Systemic Sclerosis associated Pulmonary Fibroproliferative Vasculopathy. Arthritis Rheum 63:S171-172, Abstract.

34. Wynn TA: Cellular and molecular mechanisms of fibrosis. J Pathol 2008, 214:199-210.

35. Varga J, Abraham D: Systemic Sclerosis: A prototypic multi-system fibrotic disorder. J Clin Invest 2007, 117:557-67.

36. Bhattacharyya S, Wei J, Varga J: Understanding fibrosis in systemic sclerosis: shifting paradigms, emerging opportunities. Nat Rev Rheumatol 2011.

37. Varga J, Whitfield ML: Transforming growth factor-beta in systemic sclerosis (scleroderma). Front Biosci (Schol Ed) 2009, 1:226-35.

38. Kokudo T, Suzuki Y, Yoshimatsu Y, Yamazaki T, Watabe T, Miyazono K: Snail is required for TGFbeta-induced endothelial-mesenchymal transition of embryonic stem cell-derived endothelial cells. J Cell Sci 2008, 121:3317-3324.

39. Medici D, Shore EM, Lounev VY, Kaplan FS, Kalluri R, Olsen BR: Conversion of vascular endothelial cells into multipotent stem-like cells. Nat Med 2010, 16:1400-6.

40. Li J, Qu X, Yao J, Caruana G, Ricardo SD, Yamamoto Y, Yamamoto H, Bertram JF: Blockade of endothelial-mesenchymal transition by a Smad3 inhibitor delays the early development of streptozotocin-induced diabetic nephropathy. Diabetes 2010, 59:2612-24.

41. $L i$ Z , Jimenez SA: Protein kinase $C$ delta and the $c$-abl kinase are required for transforming growth factor-beta induction of endothelialmesenchymal transition in vitro. Arthritis Rheum 2011.

42. Medici D, Potenta S, Kalluri R: Transforming growth factor-b2 promotes Snail-mediated endothelia-mesenchymal transition through convergence of Smad-dependent and Smad-independent signalling. Biochem J 2011, 438:515-520.

43. Aisagbonhi O, Rai M, Ryzhov S, Atria N, Feoktistov I, Hatzopoulos AK: Experimental myocardial infarction triggers canonical Wnt signaling and endothelial-to-mesenchymal transition. Disease Models and Mechanisms.

44. Zhu P, Huang L, Ge X, Yan F, Wu R, Ao Q: Transdifferentiation of pulmonary arteriolar endothelial cells into smooth muscle-like cells regulated by myocardin involved in hypoxia-induced pulmonary vascular remodelling. Int J Exp Pathol 2006, 87:463-74.

doi:10.1186/1755-1536-5-S1-S7

Cite this article as: Piera-Velazquez and Jimenez: Molecular mechanisms of endothelial to mesenchymal cell transition (EndoMT) in experimentally induced fibrotic diseases. Fibrogenesis \& Tissue Repair 2012 5(Suppl 1):S7. 